\title{
THE STRUCTURE OF BOUNDED BILINEAR FORMS ON PRODUCTS OF $C^{*}$-ALGEBRAS
}

\author{
KARI YLINEN
}

(Communicated by John B. Conway)

\begin{abstract}
Let $A_{1}$ and $A_{2}$ be $C^{*}$-algebras and $B: A_{1} \times A_{2} \rightarrow \mathrm{C}$ be a bounded bilinear form. It is proved that there exist a Hilbert space $H$, two Jordan morphisms $\mu_{i}: A_{i} \rightarrow L(H), i=1,2$, and two vectors $\xi_{1}, \xi_{2} \in H$ such that

$$
B(x, y)=\left(\mu_{1}(x) \xi_{1} \mid \mu_{2}\left(y^{*}\right) \xi_{2}\right) \text { for all } x \in A_{1}, y \in A_{2} .
$$
\end{abstract}

The proof depends on the Grothendieck-Pisier-Haagerup inequality and Halmos's unitary dilation theorem. An extemely elementary proof of the latter is given.

1. Introduction. In [8, Remark $5.3(\mathrm{a})]$ it was observed that some arguments in the proof of [8, Theorem 5.2] yield (essentially, cf. Remark 2.2) the following representation theorem for bounded bilinear forms on the Cartesian product of two $C^{*}$-algebras.

1.1. Lemma. Let $A_{1}$ and $A_{2}$ be two $C^{*}$-algebras and $B: A_{1} \times A_{2} \rightarrow \mathbf{C}$ be a bounded bilinear form. Then there exist four Hilbert spaces $K_{i}^{\prime}, K_{i}^{\prime \prime}, i=1,2$, cyclic *-representations $\pi_{i}^{\prime}: A_{i} \rightarrow L\left(K_{i}^{\prime}\right)$ with unit cyclic vectors $\xi_{1}^{\prime} \in K_{i}^{\prime}$, cyclic *-antirepresentations $\pi_{i}^{\prime \prime}: A_{i} \rightarrow L\left(K_{i}^{\prime \prime}\right)$ with unit cyclic vectors $\xi_{i}^{\prime \prime} \in K_{i}^{\prime \prime}$ and a bounded linear map $T: K_{1}^{\prime} \oplus K_{1}^{\prime \prime} \rightarrow K_{2}^{\prime} \oplus K_{2}^{\prime \prime}$ with $\|T\| \leq\|B\|$ such that for all $x \in A_{1}, y \in A_{2}$

$$
B(x, y)=\left(T\left(\pi_{1}^{\prime}(x) \xi_{1}^{\prime}, \pi_{1}^{\prime \prime}(x) \xi_{1}^{\prime \prime}\right) \mid\left(\pi_{2}^{\prime}\left(y^{*}\right) \xi_{2}^{\prime}, \pi_{2}^{\prime \prime}\left(y^{*}\right) \xi_{2}^{\prime \prime}\right)\right)_{K_{2}^{\prime} \oplus K_{2}^{\prime \prime}}
$$

Here and elsewhere, for any Hilbert space $H,(\cdot \mid \cdot)_{H}$ or $(\cdot \mid \cdot)$ denotes the inner product and $L(H)$ the space of bounded linear operators on $H$. If $K$ is a closed subspace of $H, P_{K}$ is the orthogonal projection of $H$ onto $K$.

The aim of this note is to point out that a representation in terms of Jordan morphisms can be obtained even without the help of the operator $T$. In the formulation of Theorem 2.1 the term "Jordan morphism" is only used for euphony; Jordan morphisms are known to be precisely the direct sums of *-representations and *-antirepresentations [12], and the proof actually yields such direct sums.

In the appendix we spell out for completeness the details of a proof of Lemma 1.1, since the proof is short and our point of view differs somewhat from that of [8]. We also find it worthwhile to communicate an utterly short and elementary proof of the Halmos dilation theorem used in the proof of Theorem 2.1.

Received by the editors November 17, 1986.

1980 Mathematics Subject Classification (1985 Revision). Primary 46L05. 


\section{The main result.}

2.1. TheOREM. Let $A_{1}$ and $A_{2}$ be $C^{*}$-algebras and $B: A_{1} \times A_{2} \rightarrow \mathrm{C}$ be a bounded bilinear form. There exist a Hilbert space $H$, two Jordan morphisms $\mu_{i}: A_{i} \rightarrow L(H), i=1,2$, and two vectors $\xi_{1}, \xi_{2} \in H$ such that

$$
B(x, y)=\left(\mu_{1}(x) \xi_{1} \mid \mu_{2}\left(y^{*}\right) \xi_{2}\right) \quad \text { for all } x \in A_{1}, y \in A_{2} \text {. }
$$

ProOF. We use Lemma 1.1 and its notation, and write $K=K_{1} \oplus K_{2}$ where $K_{i}=K_{i}^{\prime} \oplus K_{i}^{\prime \prime}, i=1,2$. We modify a technique used in the proof of Theorem 2.4 in [4]. Define $\tilde{T}: K \rightarrow K$ by the formula $\tilde{T}(u, v)=(0, T u)$. We may assume that $\|B\| \leq 1$, so that $\|\tilde{T}\| \leq 1$. Thus $\tilde{T}$ has a unitary dilation, i.e., there is a Hilbert space $H$ containing $K$ as a subspace such that $T=P_{K} U \mid K$ for some unitary operator $U: H \rightarrow H$ (see $\S 3$ for a proof and references). Write $H$ in the form $H=K_{1} \oplus K_{2} \oplus K^{\perp}$, denote $\pi_{i}=\pi_{i}^{\prime} \oplus \pi_{i}^{\prime \prime}: A_{i} \rightarrow L\left(K_{i}\right)$ for $i=1,2$, and define $\mu_{1}(x)\left(\varsigma_{1}, \varsigma_{2}, \varsigma_{3}\right)=\left(\pi_{1}(x) \varsigma_{1}, 0,0\right)$ for $x \in A_{1}, \varsigma_{1} \in K_{1}, \varsigma_{2} \in K_{2}, \varsigma_{3} \in K^{\perp}$, and $\mu_{2}(y)=U^{*}\left(0 \oplus \pi_{2}(y) \oplus 0\right) U$ for $y \in A_{2}$. Moreover, denote $\xi_{1}=\left(\left(\xi_{1}^{\prime}, \xi_{1}^{\prime \prime}\right), 0,0\right)$ and $\xi_{2}=U^{*}\left(0,\left(\xi_{2}^{\prime}, \xi_{2}^{\prime \prime}\right), 0\right)$. A direct calculation using (1) in Lemma 1.1 now gives

$$
\begin{aligned}
& \left(\mu_{1}(x) \xi_{1} \mid \mu_{2}\left(y^{*}\right) \xi_{2}\right)_{H} \\
& \quad=\left(U\left(\pi_{1}(x)\left(\xi_{1}^{\prime}, \xi_{1}^{\prime \prime}\right), 0,0\right) \mid\left(0, \pi_{2}\left(y^{*}\right)\left(\xi_{2}^{\prime}, \xi_{2}^{\prime \prime}\right), 0\right)\right)_{H} \\
& \quad=\left(\tilde{T}\left(\pi_{1}(x)\left(\xi_{1}^{\prime}, \xi_{1}^{\prime \prime}\right), 0\right) \mid\left(0, \pi_{2}\left(y^{*}\right)\left(\xi_{2}^{\prime}, \xi_{2}^{\prime \prime}\right)\right)\right)_{K} \\
& \quad=\left(T \pi_{1}(x)\left(\xi_{1}^{\prime}, \xi_{1}^{\prime \prime}\right) \mid \pi_{2}\left(y^{*}\right)\left(\xi_{2}^{\prime}, \xi_{2}^{\prime \prime}\right)\right)_{K_{2}}=B(x, y) .
\end{aligned}
$$

2.2. REMARK. Applying Theorem 2.1 to $\tilde{B}: A_{2} \times A_{1} \rightarrow \mathrm{C}$ defined by $\tilde{B}(y, x)=$ $B(x, y)$ we see that $B$ can also be represented in the form $B(x, y)=\left(\mu_{1}(x) \mu_{2}(y) \xi_{1} \mid \xi_{2}\right)$, where $\mu_{i}: A_{i} \rightarrow L(H)$ are Jordan morphisms for some Hilbert space $H$, and $\xi_{i} \in H$ for $i=1,2$. Similarly, the technique of the above proof shows that in [3, Theorem 2.1 , condition (3)], one may take $H=K$ and leave the operator $T$ out. Theorem 2.1 of the present paper was announded at the UCLA Functional Analysis seminar October 8, 1986; I am indebted to E. G. Effros for bringing a preprint of [3] in that connection to my attention.

3. Appendix: alternate proofs of essentially known results. The unitary dilation result in [7, Problem 177(a)] due to Halmos [6] is contained in part (b) of the following proposition. Unlike $[\mathbf{6}, \mathbf{7}]$ or the proof of $(\mathrm{a})$ in $[\mathbf{2}]$, the proof below does not depend on the existence of the square root of a positive operator. The proof of (a) may actually be seen as flowing from a comparison of [2] with a more traditional approach (see e.g. $[1,9-11])$ to the kind of problem treated there.

3.1. Proposition. Let $H_{1}$ and $H_{2}$ be Hilbert spaces and $T: H_{1} \rightarrow H_{2}$ a linear contraction.

(a) There exist a Hilbert space $K_{2}$ containing $H_{2}$ as a subspace, and an isometric linear map $V: H_{1} \rightarrow K_{2}$ such that $T=P_{H_{2}} V$.

(b) There exist Hilbert spaces $K_{1}$ and $K_{2}$ and an isometric linear surjection $U: K_{1} \rightarrow K_{2}$ such that $K_{i}$ contains $H_{i}$ as a subspace, $i=1,2$, and $T=P_{H_{2}} U \mid H_{1}$.

ProOF. (a) Denote $h(x, y)=(x \mid y)-(T x \mid T y)$ for $x, y \in H_{1}$. Then $h: H_{1} \times H_{1} \rightarrow$ $\mathrm{C}$ is sesquilinear, and positive since $\|T\| \leq 1$. Denote $N=\left\{x \in H_{1} \mid h(x, x)=\right.$ $0\}$, and complete $H_{1} / N$ with the inner product $(x+N \mid y+N)=h(x, y)$ to a 
Hilbert space $K$. Finally define $K_{2}=K \oplus H_{2}$, and $V x=(x+N, T x) \in K_{2}$ for $x \in H_{1}$. Interpreting $H_{2}$ as a subspace of $K_{2}$, we have $T=P_{H_{2}} V$, and $\|V x\|^{2}=\|x+N\|^{2}+\|T x\|^{2}=\|x\|^{2}$.

(b) Continuing from (a), denote $K_{1}=H_{1} \oplus\left(K_{2} \ominus V\left(H_{1}\right)\right)$, and define $U(x, y)=$ $V x+y$ for $x \in H_{1}, y \in K_{2} \oplus V\left(H_{1}\right)$.

PROOF OF LEMMA 1.1. We may assume that $\|B\| \leq 1$. Denote $B_{0}(x, y)=$ $B\left(x, y^{*}\right)$, so that $B_{0}$ is a bounded sesquilinear form with $\left\|B_{0}\right\| \leq 1$. Using Haagerup's general version [5, Theorem 1.1] of Pisier's Grothendieck type inequality we get four states $\varphi_{i}, \psi_{i}: A_{i} \rightarrow \mathbf{C}, i=1,2$, such that

$$
\left|B_{0}(x, y)\right| \leq\left[\varphi_{1}\left(x^{*} x\right)+\psi_{1}\left(x x^{*}\right)\right]^{1 / 2}\left[\varphi_{2}\left(y^{*} y\right)+\psi_{2}\left(y y^{*}\right)\right]^{1 / 2}
$$

for all $x \in A_{1}, y \in A_{2}$. (In a similar estimate for $B$ exchange the roles of the last two states.) Denoting $h_{i}(u, v)=\varphi_{i}\left(v^{*} u\right)+\psi_{i}\left(u v^{*}\right)$ and $N_{i}=\{u \in$ $\left.A_{i} \mid h_{i}(u, u)=0\right\}$, we get two inner product spaces $A_{i} / N_{i}$ with the inner products $\left(u+N_{i} \mid v+N_{i}\right)=h_{i}(u, v)$. Completing these to Hilbert spaces $K_{i}$ we obtain a well-defined bounded sesquilinear form $\tilde{B}_{0}: K_{1} \times K_{2} \rightarrow \mathrm{C}$ with $\left\|\tilde{B}_{0}\right\| \leq 1$ and $\tilde{B}_{0}\left(x+N_{1}, y+N_{2}\right)=B_{0}(x, y), x \in A_{1}, y \in A_{2}$. Thus there is a bounded linear operator $\tilde{T}: K_{1} \rightarrow K_{2}$ such that $\tilde{B}_{0}(w, z)=(\tilde{T} w \mid z), w \in K_{1}, z \in K_{2}$. On the other hand, applying the GNS-construction [13, Theorem 9.14] to $A_{i}$ and $\varphi_{i}$, and to the opposite $C^{*}$-algebra (i.e., otherwise the same as $A_{i}$ but equipped with the product $u \cdot v=v u$ where $v u$ is the product of $A_{i}$ ) and $\psi_{i}$ we see that $\varphi_{i}(u)=\left(\pi_{i}^{\prime}(u) \xi_{i}^{\prime} \mid \xi_{i}^{\prime}\right)$ and $\psi_{i}(u)=\left(\pi_{i}^{\prime \prime}(u) \xi_{i}^{\prime \prime} \mid \xi_{i}^{\prime \prime}\right)$ for a cyclic *-representation $\left\{\pi_{i}^{\prime}, K_{i}^{\prime}, \xi_{i}^{\prime}\right\}$ and a cyclic *-antirepresentation $\left\{\pi_{i}^{\prime \prime}, K_{i}^{\prime \prime}, \xi_{i}^{\prime \prime}\right\}$ of $A_{i}$. A straightforward calculation shows that $\left(u+N_{i} \mid v+N_{i}\right)$ is the same as the inner product of $\left(\pi_{i}^{\prime}(u) \xi_{i}^{\prime}, \pi_{i}^{\prime \prime}(u) \xi_{i}^{\prime \prime}\right)$ and $\left(\pi_{i}^{\prime}(v) \xi_{i}^{\prime}, \pi_{i}^{\prime \prime}(v) \xi_{i}^{\prime \prime}\right)$ in $K_{i}^{\prime} \oplus K_{i}^{\prime \prime}$, which implies that we get for each $i=1,2$ a well-defined isometric linear map $V_{i}: K_{i} \rightarrow K_{i}^{\prime} \oplus K_{i}^{\prime \prime}$ satisfying $V_{i}\left(u+N_{i}\right)=\left(\pi_{i}^{\prime}(u) \xi_{i}^{\prime}, \pi_{i}^{\prime \prime}(u) \xi_{i}^{\prime \prime}\right)$ for $u \in A_{i}$. It is now easy to verify that the choice $T=V_{2} \tilde{T} V_{1}^{*}$ will work.

\section{REFERENCES}

1. J. L. Abreu, A note on harmonizable and stationary sequences, Bol. Soc. Mat. Mexicana 15 (1970), 48-51.

2. S. D. Chatterji, Orthogonally scattered dilation of Hilbert space valued set functions, Measure Theory (Proc. Conf. Oberwolfach, 1981), Lecture Notes in Math., vol. 945, Springer-Verlag, Berlin and New York, 1982, pp. 269-281.

3. E. G. Effros and A. Kishimoto, Module maps and Hochschild-Johnson cohomology, Indiana Univ. J. Math. 36 (1987), 257-276.

4. C. C. Graham and B. M. Schreiber, Bimeasure algebras on LCA groups, Pacific J. Math. 115 (1984), 91-127.

5. U. Haagerup, The Grothendieck inequality for bilinear forms on $C^{*}$-algebras, Advances in Math. 56 (1985), 93-116.

6. P. R. Halmos, Normal dilations and extensions of operators, Summa Brasil. Math. 2 (1950), 125-134.

7. —_, A Hilbert space problem book, Van Nostrand, Toronto, Princeton, N.J., and London, 1967.

8. S. Kaijser and A. M. Sinclair, Projective tensor products of $C^{*}$-algebras, Math. Scand. 55 (1984), 161-187.

9. A. G. Miamee and H. Salehi, Harmonizability, V-boundedness and stationary dilation of stochastic processes, Indiana Univ. Math. J. 27 (1978), 37-50. 
10. H. Niemi, On stationary dilations and the linear prediction of certain stochastic processes, Soc. Sci. Fenn. Comment. Phys.-Math. 45 (1975), 111-130.

11. $\ldots$, On orthogonally scattered dilations of bounded vector measures, Ann. Acad. Sci. Fenn. AI Math. 3 (1977), 43-52.

12. E. Størmer, On the Jordan structure of $C^{*}$-algebras, Trans. Amer. Math. Soc. 120 (1965), 438-447.

13. M. Takesaki, Theory of operator algebras. I, Springer-Verlag, New York, Heidelberg and Berlin, 1979.

DePARTMENT OF MATHEMATICS AND COMPUTER SCIENCE, UNiversity OF CALIFORNIA, RIVERSIDE, CALIFORNIA 92521-0135

DEPARTMENT OF MATHEMATICS, UNIVERSity OF TURKU, SF-20500 TURKU, FinLAND (Current address) 